\title{
A Study of Notations and Illustrations of Axiomatic Fuzzy Set Theory
}

\author{
Lakshmi Ramani Burra \\ Research Scholar \\ GITAM University \\ Visakhapatnam, India
}

\author{
Padmaja Poosapati \\ Associate Professor \\ GITAM University \\ Visakhapatnam, India
}

\begin{abstract}
Fuzzy logic system studies reasoning systems in which the design of precision and deception are considered in a graded fashion, in contrast with classical mathematics where only absolutely true statements are considered. Whereas, Axiomatic fuzzy logic system facilitates a significant step on how to transform the information within databases into the membership functions and their fuzzy logic operations, by taking both the fuzziness and randomness into account. In this paper, various notations and illustrations of fuzzy concepts and coherence membership functions have been studied and analyzed under the framework of Axiomatic Fuzzy set theory. Various examples are illustrated for every concept by considering the hypothetical data.
\end{abstract}

\section{Keywords}

Axiomatic Fuzzy Set structures, Axiomatic Fuzzy Set algebras, Axiomatic Fuzzy Set logic, Coherence membership functions, Fuzzy logic system.

\section{INTRODUCTION}

In today's enormous storage era, knowledge acquisitions and representations constitute a major knowledge engineering bottleneck. There are various methods intended at improving this problem [1]. The unification of fuzzy sets into the representations of fuzzy concepts makes it possible for us to combine the uncertainty handling and approximate reasoning capabilities with comprehensibility. In conventional fuzzy theories, the membership functions of fuzzy sets are often given by personal intuition manually and the logic operations are equipped by a kind of triangular norms as t-norms, $\mathrm{t}$ conorms and s-norms which is chosen in advance and independent of the distribution of the raw data [2]. The largescale intelligence systems in real-world applications are usually very large and complex, containing such a large number of concepts that it is impossible or difficult to define the membership functions just by expert perception [3].

In order to deal with the above issues, the authors of papers [1-4] have proposed Axiomatic Fuzzy Set (AFS) theory in which fuzzy sets membership functions and their logic operations are impersonally and automatically determined according to the distributions of original data and the semantics of the fuzzy concepts. In understanding the nature of learning approach the complement operation of the fuzzy sets has been given, thus a fuzzy logic system, which is called AFS fuzzy logic, has been developed [2]. In recent times, AFS theory has been developed and applied to fuzzy decision trees [5], fuzzy clustering analysis [6], fuzzy classifier [7, 8], formal concept analysis

[9], fuzzy rough sets [10], fuzzy cognitive maps [11] and credit rating analysis [12], etc.
In AFS theory, the membership functions and their logic operations are determined by AFS structures and AFS algebras. An AFS structure is a mathematical object of triple $(M, \tau, X)$ which is a special family of combinatorics objects [8], where $X$ is the set of objects, $M$ is a set of some simple concepts [13] on $X$ and $\tau$ is mathematical abstract of the complex relations of objects concerning the original data and facts such as database sub-preference relations [13], even human intuition descriptions. AFS algebra is a family of molecular lattices (i.e. completely distributive lattices) generated by sets such as $X, M$. With the AFS structure $(M, \tau, X)$, which can be directly obtained from the given dataset, any concepts on $X$ can be represented by the fuzzy sets in $E M$, which is the AFS algebra over $M$. Using AFS algebra and AFS structure $(M, \tau, X)$, a great large number of complex fuzzy concepts on $X$ and their logic operations can be implemented by the algebraic operators on few simple concepts in $M$. The membership functions and their logic operations are more accurate and impersonal reflections of the original data and facts than given by human intuition in other fuzzy theories, and the information contained in the original data and facts is preserved at major extent [3].

\section{LITERATURE SURVEY}

\subsection{A review of the Axiomatic Fuzzy Set theory}

In this section, were call the notations and some of the definitions of AFS theory which has been involved many mathematical theories. It is known that the AFS theory provides an effective tool to convert the information into the training examples and databases into the membership functions and their fuzzy logic operations by taking both the subjective imprecision and objective uncertainty into consideration [14-21]. AFS theory is based on AFS structure a kind of mathematical description of the data structure, which is a special kind of combinatorics systems and AFS algebra - a kind of semantic methodology which is a family of completely distributive lattices.

\subsubsection{Axiomatic Fuzzy Set Algebras}

AFS algebras is defined as a family of completely distributive lattices and used to study the lattice value representations of fuzzy concepts.

\section{Definition 1([1])}

Let $X_{1}, X_{2}, \ldots, X_{n}, M$ be a non-empty set. Then the set $E X_{1} X_{2} \ldots . X_{n} M$ is defined as

$$
\begin{aligned}
& E M^{\#}= \\
& \left\{\sum_{i \in I}\left(\prod_{m \in A_{i}} m\right) \mid A_{i} \subseteq M, i \in I, I \text { is a nonempty index set }\right\}
\end{aligned}
$$

where $\Sigma$ and $\Pi$ denotes a disjunction and conjunction. For $\sum_{i \in I}\left(\prod_{m \in A_{i}} m\right)$, which is a formal sum of $\left(\prod_{m \in A_{i}} m\right), A_{i} \subseteq$ 
$M, i \in I$, is the disjunctions of conjunctions represented by $\prod_{m \in A i} m^{\prime} s$ (i.e., the disjunctive normal form a formula representing a concept).

\section{Example 1}

Let $X=\left\{p_{1}, p_{2}, p_{3}, p_{4}, p_{5}\right\}$ be a set of 5 persons, $M=$ $\left\{m_{1}, m_{2}, \ldots, m_{8}\right\}$ be a set of attributes where $m_{1}=$ Bengali, $m_{2}=$ Marathi, $m_{3}=$ Assame, $m_{4}=$ male, $m_{5}=$ female, $m_{6}=$ Professor, $m_{7}=$ Engineer, $m_{8}=$ Doctor, i.e., each feature/attribute is a crisp concept are shown in table 1.

Table 1: Crisp attributes descriptions

\begin{tabular}{|c|c|c|c|c|c|c|c|c|}
\hline & Bengali & Marathi & Assame & Male & Female & Prof. & Eng. & Doctor \\
\hline$p_{1}$ & $\mathrm{Y}$ & $\mathrm{N}$ & $\mathrm{N}$ & $\mathrm{Y}$ & $\mathrm{N}$ & $\mathrm{N}$ & $\mathrm{Y}$ & $\mathrm{Y}$ \\
\hline$p_{2}$ & $\mathrm{~N}$ & $\mathrm{Y}$ & $\mathrm{N}$ & $\mathrm{N}$ & $\mathrm{Y}$ & $\mathrm{Y}$ & $\mathrm{N}$ & $\mathrm{N}$ \\
\hline$p_{3}$ & $\mathrm{~N}$ & $\mathrm{Y}$ & $\mathrm{N}$ & $\mathrm{Y}$ & $\mathrm{N}$ & $\mathrm{N}$ & $\mathrm{Y}$ & $\mathrm{N}$ \\
\hline$p_{4}$ & $\mathrm{Y}$ & $\mathrm{N}$ & $\mathrm{N}$ & $\mathrm{Y}$ & $\mathrm{N}$ & $\mathrm{Y}$ & $\mathrm{N}$ & $\mathrm{N}$ \\
\hline$p_{5}$ & $\mathrm{~N}$ & $\mathrm{~N}$ & $\mathrm{Y}$ & $\mathrm{Y}$ & $\mathrm{N}$ & $\mathrm{Y}$ & $\mathrm{Y}$ & $\mathrm{N}$ \\
\hline
\end{tabular}

For example, $\gamma=m_{1} m_{5}+m_{3} m_{8}+m_{6}$ this translates as "bengali female" or "Assame doctor" or "professor". (the "+" denotes here a disjunction of concepts) while $M$ may be a set of fuzzy or Boolean (two - valued) concepts, every $\sum_{i \in I}\left(\prod_{m \in A_{i}} m\right), \mid \mathrm{A}_{\mathrm{i}} \subseteq M, i \in I$ has a well-defined meaning.

\section{Definition 2 ([1])}

Let $M$ be a non-empty set. A binary relation $R$ on $E M$ is defined as follows:

$$
\begin{aligned}
& \text { for, } \sum_{i \in I}\left(\prod_{m \in A_{i}} m\right), \sum_{j \in J}\left(\prod_{m \in B_{j}} m\right) \in E M \\
& \text { and }\left[\sum_{i \in I}\left(\prod_{m \in A_{i}} m\right)\right] R\left[\sum_{j \in J}\left(\prod_{m \in B_{j}} m\right)\right] \Leftrightarrow
\end{aligned}
$$

(i) $\forall \mathrm{A}_{\mathrm{i}}(\mathrm{i} \in \mathrm{I}), \exists \mathrm{B}_{\mathrm{h}}(\mathrm{h} \in \mathrm{J})$ such that $\mathrm{A}_{\mathrm{i}} \subseteq \mathrm{B}_{\mathrm{h}}$

(ii) $\forall \mathrm{B}_{\mathrm{j}}(\mathrm{j} \in \mathrm{J}), \exists \mathrm{A}_{\mathrm{k}}(\mathrm{k} \in \mathrm{I})$ such that $\mathrm{B}_{\mathrm{j}} \subseteq \mathrm{A}_{\mathrm{k}}$

It is obvious that $R$ is an equivalence relation. The quotient set $E M^{\#} / R$ is represented by $E M$.

The notation,

$\sum_{i \in I}\left(\prod_{m \in A i} m\right)=\sum_{i \epsilon J}\left(\prod_{m \in B j} m\right)$ means that $\sum_{i \epsilon I}\left(\prod_{m \in A i} m\right)$ and $\sum_{i \epsilon J}\left(\prod_{m \in B j} m\right)$ are equivalent under equivalence relation $R$. Thus the semantics they represent are equivalent.

In example 1, for $\xi=m_{3} m_{8}+m_{1} m_{4}+m_{2} m_{5} m_{7}+$ $m_{1} m_{4} m_{8}$

$$
\zeta=m_{3} m_{8}+m_{1} m_{4}+m_{2} m_{5} m_{7} \in E M
$$

By definition 2, this implies that $\xi=\zeta$.

\section{Theorem 1([1])}

Let $M$ be a non-empty set. Then $(E M, \vee, \wedge)$ forms a completely distributive lattice under the binary compositions $\checkmark$ and $\Lambda$ defined as follows.

For any, $\sum_{i \in I}\left(\prod_{m \in A_{i}} m\right), \sum_{j \in J}\left(\prod_{m \in B_{j}} m\right) \in E M$.

$$
\begin{gathered}
\sum_{i \in I}\left(\prod_{m \in A_{i}} m\right) \vee\left(\sum_{j \in J}\left(\prod_{m \in B_{j}} m\right)=\sum_{k \in I \cup \mathrm{J}}\left(\prod_{m \in C_{k}} m\right)\right. \\
\sum_{i \in I}\left(\prod_{m \in A_{i}} m\right) \wedge\left(\sum_{j \in J}\left(\prod_{m \in B_{j}} m\right)=\right. \\
\sum_{i \in \mathrm{I}, \mathrm{j} \in J}\left(\prod_{m \in A_{i} \cup B_{\mathrm{j}}} m\right),
\end{gathered}
$$

where for any $k \in I \cup J$ (the disjoint union of $I$ and $J$, i.e., every element in $I$ and every element in $J$ are always regarded as different elements in $I \cup J), C_{k}=A_{k}$ if $k \in I$, and $C_{k}=$ $B_{k}$ if $k \in J$.

For example,

The new attributes $\alpha, \beta$ and $\gamma$ can be represented by the elements in EM as $\alpha=\left\{m_{1} m_{4}\right\}+\left\{m_{2} m_{5} m_{6}\right\}$,

$$
\begin{aligned}
& \beta=\left\{m_{1} m_{4}\right\}+\left\{m_{2} m_{5} m_{6}\right\}+\left\{m_{1} m_{4} m_{8}\right\} \text { and } \\
& \gamma=\left\{m_{5} m_{6}\right\}+\left\{m_{5} m_{8}\right\}
\end{aligned}
$$

$\alpha=\left(m_{1} m_{4}\right)+\left(m_{2} m_{5} m_{6}\right)$ which translates the meaning that: "Bengali males or Marathi female professors";

$\beta=\left(m_{1} m_{4}\right)+\left(m_{2} m_{5} m_{6}\right)+\left(m_{1} m_{4} m_{8}\right)$ which translates the meaning that: "Bengali males or Marathi female professors or Bengali male Doctors";

$\gamma=\left(m_{5} m_{6}\right)+\left(m_{5} m_{8}\right)$ which translates the meaning that: "Female Professors or Female Doctors".

In general, $M$ is a set of fuzzy or crisp attributes, for $\sum_{i \in I} A_{i} \in$ $E M, \mathrm{~A}_{\mathrm{i}}$ expresses a new attribute generated by the "and", "or" of all attributes in $A_{i}, i \in I$. Even if there are fuzzy attributes in $M, \sum_{i \in I} A_{i}$ has definite semantic signification like crisp attributes $\alpha, \beta$ and $\gamma$ which are discussed above. Representing attributes generated by the attributes in $M$ in the form $\sum_{i \in I} A_{i} \in E M$ can not only preserve the semantic signification, but also avoid to define the fuzzy logic operators "and", "or" in advance.

By Definition 2,

$$
\begin{gathered}
\beta=\left(\mathrm{m}_{1} \mathrm{~m}_{4}\right)+\left(\mathrm{m}_{2} \mathrm{~m}_{5} \mathrm{~m}_{6}\right)+\left(\mathrm{m}_{1} \mathrm{~m}_{4} \mathrm{~m}_{8}\right) \\
=\left(m_{1} m_{4}\right)+\left(m_{2} m_{5} m_{6}\right)=\alpha
\end{gathered}
$$

From Table 1 , it verifies that for $x \in X, x$ satisfies $\alpha$ if and only if it satisfies $\beta$. In other words, term $\left\{m_{1} m_{4} m_{8}\right\}$ in $\beta$ is redundant. In general, $M$ is a set of fuzzy or crisp concepts, for $\alpha, \beta \in E M$. If $\alpha=\beta$, then the ordinary fuzzy sets (i.e., the membership degrees in the interval $[0,1]$ ) or L-fuzzy sets (i.e., the membership degrees in a lattice L) representing fuzzy concepts $\alpha$ and $\beta$ should be identical. In the following, each element in $E M$ denote it as a fuzzy concept or crisp concept. The semantic significations of concepts " $\alpha$ or $\gamma$ " and " $\alpha$ and $\gamma$ " can be simply denoted as $\alpha \vee \gamma$ and $\alpha \wedge \gamma$ respectively with (1), (2), therefore

$$
\begin{gathered}
\alpha \vee \gamma=\left\{m_{1} m_{4}\right\}+\left\{m_{2} m_{5} m_{6}\right\}+\left\{m_{5} m_{6}\right\}+\left\{m_{5} m_{8}\right\} \\
=\left\{m_{5} m_{6}\right\}+\left\{m_{5} m_{8}\right\}+\left\{m_{1} m_{4}\right\} \\
\alpha \vee \gamma=\left\{m_{1} m_{4}\right\}+\left\{m_{2} m_{5} m_{6}\right\}+\left\{m_{5} m_{6}\right\}+\left\{m_{5} m_{8}\right\} \\
=\left\{m_{5} m_{6}\right\}+\left\{m_{5} m_{8}\right\}+\left\{m_{1} m_{4}\right\}
\end{gathered}
$$

In general, for any fuzzy concepts $\alpha, \beta \in E M$, to define their membership functions and fuzzy logic operators, fuzzy concepts $\alpha \vee \beta$ and $\alpha \wedge \beta$ are the fuzzy logic "or", "and" of $\alpha, \beta$, respectively. $\alpha \geq \beta$ in lattice EM implies that no matter how to define the membership functions for $\alpha, \beta$, the membership degree of any $x$ belonging to $\alpha$ should be larger 
than or equal to that of $x$ belonging to $\beta$. For $\mathrm{M}$ a set of few fuzzy or crisp concepts, a great number of fuzzy concepts can be expressed by the elements in $E M$ and the fuzzy logic operations of them can be implemented by the operations $\vee, \wedge$ of the completely distributive lattice $(E M, \vee, \wedge)$.

\subsubsection{Axiomatic Fuzzy Set Structures}

In this work, the fuzzy set of $X$ denote that all kinds of representing forms for fuzzy concepts. The fuzzy sets and crisp subsets on $X$ can be explained as the following:

For a fuzzy set $\zeta$ on universe of discourse $X$, any $x \in X$, either $\mathrm{x}$ belongs to $\zeta$ at some degree or does not belong to $\zeta$ at all. While for a crisp subset $A$ of $X$, any $x \in X$, either $x$ belongs to $A$ or does not belong to $A$ at all. Based on this proclamation that, both a fuzzy set and a crisp subset on $X$ can be represented by a binary relation $R$ on $X$ through comparing the degrees of each pair $x, y$ in $X$ belonging to the concept without require to classify their membership degrees.

\section{Definition 3([1])}

Let $\zeta$ be any concept on the universe of discourse $X . R_{\zeta}$ is called a binary relation (i.e., $R_{\zeta} \subset \mathrm{X} \times \mathrm{X}$ ) of $\zeta$ if $R_{\zeta}$ satisfies: $x, y \in X,(x, y) \in R_{\zeta} \Leftrightarrow x$ belongs to concept $\zeta$ at some degree and the degree of $x$ belonging to $\zeta$ is larger than or equals to that of $y$, or $x$ belongs to concept $\zeta$ at some degree and $y$ does not at all.

Even though fuzzy concepts are indistinct, the binary relations corresponding to them are crisp subset of $X \times X$. And the notation that $(x, x) \in R_{\zeta}$ means that $x$ belongs to concept $\zeta$ at some degree and $(x, x) \notin R_{\zeta}$ means that does not belong to concept at all.

\section{Definition 4([14])}

Let $X$ be a set and $R$ be a binary relation on $X . R$ is called a preference relation on $X$ if it satisfies the following conditions:
1. $\forall x \in X,(x, x) \in R$.
2. if $(x, y) \in R,(y, z) \in R$, then $(x, z) \in R, x, y, z \in X$.
3. for any $x, y \in X$, either $(x, y) \in R$ or $(y, x) \in R$.

Although preference relations are very simple and have very good mathematical properties, it is too strict to represent ordinary concepts. So, it can be given that the following definition, which is mathematical abstraction of the simple concepts

\section{Definition 5 ([14])}

Let $X$ be a set and $R$ be a binary relation on $X . R$ is called a sub-preference relation on $X$ if for $x, y, z \in X, x=y$, $R$ satisfies the following conditions:

1. if $(x, y) \in R$, then $(x, x) \in R$;

2. if $(x, x) \in R$ and $(y, y) \notin R$, then $(x, y) \in$ $R$; if $(x, y),(y, z) \in R$, then $(x, z) \in R$;

3. if $(x, x) \in R$ and $(y, y) \in R$, then either $(x, y) \in R$ or

$$
(y, x) \in R \text {. }
$$

A concept $\zeta$ is called simple concept on $X$ if $R_{\zeta}$ is a subpreference relation. Otherwise $\zeta$ is called complex concept on $\mathrm{X}$.

\section{Definition 6 ([14])}

Let $m$ be a simple concept on $X$ and $\rho_{m}: X \rightarrow R^{+}=[0, \propto)$. $\rho_{m}$ is called a weighted function of simple concept $m$ if $\rho_{m}$ satisfies the following:

1. $\rho_{m}=0 \Leftrightarrow(x, x) \notin R_{m}, x \in X$;

2. $(x, y) \in R_{m} \Rightarrow \rho_{m}(x) \geq \rho_{m}(y), x, y \in X$

The sequence systems express the information by information-based criteria such as human perception-based information, gain ratio, symmetric uncertainty, and order are regularized to be AFS structures by two axioms.

\section{Definition 7([1, 17, 23, 24])}

Let $X, M$ be two sets and $2^{M}$ be the power set of $M \tau: X \times$ $X \rightarrow 2^{M} .(M, \tau, X)$ is called an AFS structure if $\tau$ satisfies the following conditions:

$$
\begin{aligned}
& A X 1: \forall\left(x_{1}, x_{2}\right) \in X \times X, \tau\left(x_{1}, x_{2}\right) \subseteq \tau\left(x_{1}, x_{1}\right) ; \\
& \text { and } \\
& \qquad \begin{array}{r}
A X 2: \forall\left(x_{1}, x_{2}\right),\left(x_{2}, x_{3}\right) \in X \times X, \tau\left(x_{1}, x_{2}\right) \cap \tau\left(x_{2}, x_{3}\right) \\
\subseteq
\end{array}
\end{aligned}
$$

In addition, $X$ is called universe of discourse, $M$ is called an attribute set and $\tau$ is called a structure.

Let $X$ be a data set of objects and $M$ be a set of simple concepts on $X$, for any $x, y \in X, \tau(x, y)$ are subsets of $M$, $\tau(x, y)=\left\{m \mid m \in M,(x, y) \in R_{m}\right\}$, where $R_{m}$ is the binary relation of simple concept $m$ i.e., for any $m \in \tau(x, y)$, the degree of $x$ belonging to the concept $m$ is greater than or equal to y i.e., $\gamma_{\mathrm{m}}(x) \geq \gamma_{\mathrm{m}}(y)$.

\section{Example 2}

Let $X=\left\{x_{1}, x_{2}, \ldots, x_{10}\right\}$ be a set of 10 persons and $M=$ $\left\{m_{1}, m_{2}, \ldots, m_{10}\right\}$ be the set of fuzzy concepts on $X$ and the elements of $M$ are analyzed as simple concepts, where $m_{1}=$ age, $m_{2}=$ high height, $m_{3}=$ weight, $m_{4}=$ high salary, $m_{5}=$ more wealth, $m_{6}=$ male, $m_{7}=$ female, $m_{8}=$ Eyecolor black, $m_{9}=$ Eyecolorblue, $m_{10}=$ Eyecolor brown, where the attributes are described by real numbers such as age, height, weight, salary, wealth and gender is described as Boolean values and the eye color such as black, blue, brown are described as in order relations i.e., $x=y$ in the order relation implies that the degrees of $x$ and $y$ belonging to the attribute are equal, as a substitute of $x$ and $y$ being the same element of $X$.The samples $x_{1}, x_{2}, \ldots, x_{10}$ are shown in Table 2 .

From Table 2, it can verify that each concept $m \in M$ is a simple concept and the structure $\tau$ can be defined as follows.

For any $x_{i}, x_{j} \in \mathrm{X}, i \neq j$

$\tau\left(x_{i}, x_{j}\right)=$

$\left\{m / m M, x_{i}\right.$ possesses attribute $m$ at some degree $\}$

$\tau\left(x_{i}, x_{j}\right)=\left\{m / m \in M, x_{i} \geq_{m} x_{j}\right\}$

where $x_{i} \geq_{m} x_{j}$ implies that $x_{i}$ possesses attribute $m$ in some degree and the degree of $x_{i}$ belonging to $m$ is larger than or equal to that of $x_{j}$. 
For example,

$$
\begin{gathered}
\tau\left(x_{1}, x_{1}\right)=\left\{m_{1}, m_{2}, m_{3}, m_{4}, m_{6}, m_{8}, m_{9}, m_{10}\right\} \\
\tau\left(x_{4}, x_{7}\right)=\left\{m_{1}, m_{2}, m_{6}, m_{9}, m_{10}\right\}
\end{gathered}
$$

Eye color black

$m_{8}: x_{4}>x_{1}>x_{3}=x_{7}>x_{2}=x_{9}>x_{6}>x_{5}=x_{8}=x_{10} ;$

For example, the numbers in the column "Eye color" implies the order relation as $(>)$

Table 2: Representation of the samples with the attribute values

\begin{tabular}{|c|c|c|c|c|c|c|c|c|c|c|}
\hline $\begin{array}{c}\text { Sample } \\
\mathbf{X}\end{array}$ & $\begin{array}{c}\text { Age } \\
\boldsymbol{m}_{\mathbf{1}}\end{array}$ & $\begin{array}{c}\text { Height } \\
\boldsymbol{m}_{\mathbf{2}}\end{array}$ & $\begin{array}{c}\text { Weight } \\
\boldsymbol{m}_{\mathbf{3}}\end{array}$ & $\begin{array}{c}\text { Salary } \\
\boldsymbol{m}_{\mathbf{4}}\end{array}$ & $\begin{array}{c}\text { Wealth } \\
\boldsymbol{m}_{\mathbf{5}}\end{array}$ & $\begin{array}{c}\text { Male } \\
\boldsymbol{m}_{\mathbf{6}}\end{array}$ & $\begin{array}{c}\text { Female } \\
\boldsymbol{m}_{\mathbf{7}}\end{array}$ & $\begin{array}{c}\text { Eye } \\
\text { color } \\
\text { black } \\
\boldsymbol{m}_{\mathbf{8}}\end{array}$ & $\begin{array}{c}\text { Eye color } \\
\text { blue } \\
\boldsymbol{m}_{\mathbf{9}}\end{array}$ & $\begin{array}{c}\text { Eye color } \\
\text { brown } \\
\boldsymbol{m}_{\mathbf{1 0}}\end{array}$ \\
\hline$x_{1}$ & 30 & 1.9 & 90 & 1 & 0 & $y$ & $n$ & 2 & 5 & 3 \\
\hline$x_{2}$ & 20 & 1.3 & 32 & 0 & 1 & $n$ & $y$ & 4 & 3 & 1 \\
\hline$x_{3}$ & 54 & 1.6 & 67 & 200 & 35 & $n$ & $y$ & 3 & 4 & 2 \\
\hline$x_{4}$ & 83 & 1.7 & 73 & 20 & 80 & $y$ & $n$ & 1 & 6 & 4 \\
\hline$x_{5}$ & 34 & 1.4 & 54 & 15 & 2 & $y$ & $n$ & 6 & 1 & 4 \\
\hline$x_{6}$ & 37 & 1.6 & 80 & 80 & 28 & $n$ & $y$ & 5 & 2 & 2 \\
\hline$x_{7}$ & 45 & 1.7 & 78 & 350 & 89 & $y$ & $n$ & 3 & 4 & 2 \\
\hline$x_{8}$ & 70 & 1.65 & 70 & 30 & 48 & $y$ & $n$ & 6 & 1 & 4 \\
\hline$x_{9}$ & 60 & 1.82 & 83 & 25 & 100 & $n$ & $y$ & 4 & 3 & 1 \\
\hline$x_{10}$ & 10 & 1.12 & 21 & 0 & 0 & $n$ & $y$ & 6 & 1 & 4 \\
\hline
\end{tabular}

Eye color blue

$m_{9}: x_{5}=x_{8}=x_{10}>x_{6}>x_{2}=x_{9}>x_{3}=x_{7}>x_{1}>$

$x_{4}$;

Eye color brown

$m_{10}: x_{2}=x_{9}>x_{3}=x_{6}=x_{7}>x_{1}>x_{4}=x_{5}=x_{8}=x_{10}$

In this order, $x_{i}>x_{j}$ implies that the eye color of $x_{i}$ is closer to the eye color of black than the eye color of an individual $x_{j}$ . Similarly $x_{i}=x_{j}$ implies that the eye color of $x_{i}$ is same as the eye color of $x_{j}$.

The AFS structure $(M, \tau, X)$ is the mathematical abstraction of the complex relationships between universe of discourse $X$ and the attribute set $M$. This implies that the information contained in Table 2 are represented by $(M, \tau, X)$.

\section{COHERENCE MEMBERSHIP FUNCTIONS OF FUZZY SETS}

The membership functions are consistent with both the AFS logic system $(M, \vee, \wedge)$ in the sense of the underlying semantics and the distribution of the data.

\section{Definition 8 ([1])}

Let $(M, \tau, X)$ be an AFS structure of a data set $X$. For $x \in$ $X, A \subseteq M$, the set $A^{\tau}(x) \subseteq X$ is defined as follows.

$$
\begin{aligned}
& A^{\tau}(x)=\{y \mid y \in X, \tau(x, y) \supseteq A\} \\
& \quad=\left\{y \in X \mid x \geq_{m} y \text { for any } m \in A\right\}
\end{aligned}
$$

$A^{\tau}(x)$ is the set of all elements in data set $X$ whose degree of elements are belonging to concept $\prod_{m \in A i} m$ are less than or equal to $x$.

For instance, by comparing the features values of $x_{3}$ and other samples are shown in Table 2.

$$
\left\{m_{1}\right\}^{\tau}\left(x_{3}\right)=\left\{x_{1}, x_{2}, x_{3}, x_{5}, x_{6}, x_{7}, x_{10}\right\}
$$

$$
\begin{aligned}
& \left\{m_{3}\right\}^{\tau}\left(x_{3}\right)=\left\{x_{2}, x_{3}, x_{5}, x_{10}\right\} \\
& \left\{m_{1}, m_{3}\right\}^{\tau}\left(x_{3}\right)=\left\{x_{2}, x_{3}, x_{5}, x_{10}\right\}
\end{aligned}
$$

For $\zeta \in \mathrm{EM}$, let $\mu_{\zeta}: X \rightarrow[0,1]$ be the membership function of the concept $\zeta .\left\{\mu_{\zeta}(\mathrm{x}) \mid \zeta \in \mathrm{EM}\right\}$ is called a set of coherence membership functions of the AFS fuzzy logic system (EM, $\mathrm{V}$, $\wedge)$ and the AFS structure $(M, \tau, X)$, if the following conditions are satisfied.

1. for $\alpha, \beta \in E M$, if $\alpha \leq \beta$ in lattice $(E M, \wedge, \vee)$, then $\mu_{\alpha}(x) \leq \mu_{\beta}(x)$ for any $x \in X$;

2. for $x \in X, \eta=\sum_{i \in I}\left(\prod_{m \in A_{i}} m\right) \in E M$, if $A_{i}^{\tau}(x)=$ $\emptyset$ for all $i \in I$ then $\mu_{\eta}(x)=0$;

3. for $x, y \in X, A \subseteq M, \eta=\prod_{m \in A} m \in E M$, if $A^{\tau}(x) \subseteq$ $A^{\tau}(y)$, then $\mu_{\eta}(x) \leq \mu_{\eta}(y) ;$ if $A^{\tau}(x)=X$ then $\mu_{\eta}(x)=$

\section{Proposition 1}

Let $M$ be a set of simple concepts on $X$ and $(M, \tau, X)$ be an AFS structure defined as in definition 8. Let $\left\{\mu_{\zeta}(\mathrm{x}) \mid \zeta \in E M\right\}$ be a set of coherence membership functions of $(\mathrm{EM}, \mathrm{V}, \Lambda)$ and algebraic structure $(M, \tau, X)$. Then for any $\delta, \beta \in E M$, any $x \in X$,

$$
\begin{aligned}
& \mu_{\alpha \vee \beta}(x) \geq \max \left\{\mu_{\alpha}(x), \mu_{\beta}(x)\right\}, \\
& \mu_{\alpha \wedge \beta}(x) \leq \min \left\{\mu_{\alpha}(x), \mu_{\beta}(x)\right\}
\end{aligned}
$$

\section{Proof:}

In lattice (EM, $\vee, \wedge$ ), for any $\alpha, \beta \in E M$, the $\alpha \vee \beta \geq \alpha$, $\alpha \vee \beta \geq \beta$ and $\alpha \wedge \beta \leq \alpha, \alpha \wedge \beta \leq \beta$. By using the condition 1 of Definition 8 , for any $x \in X$, one has

$$
\begin{aligned}
& \mu_{\alpha \vee \beta}(x) \geq \mu_{\alpha}(x), \mu_{\alpha \wedge \beta}(x) \geq \mu_{\beta}(x), \\
& \mu_{\alpha \wedge \beta}(x) \leq \mu_{\alpha}(x), \mu_{\alpha \wedge \beta}(x) \leq \mu_{\beta}(x)
\end{aligned}
$$

This implies that the inequality is proved. 


\section{Theorem 2}

Let $M$ be a set of simple concepts on data set $X$ and $(M, \tau, X)$ be an AFS structure of a data set $X$. Let $S$ be a $\sigma$-algebra over $X$ such that for any $m \in M$ and any $x \in X,\{m\}^{\tau}(x) \in S$. For each simple concept $\gamma \in \mathrm{M}$, let $\mathcal{M}_{\gamma}$ be a measure over $S$ with $0 \leq \mathcal{M}_{\gamma}(\mathrm{U}) \leq 1$ for all $U \in S$ and $\mathcal{M}_{\gamma}(\mathrm{X})=1 .\left\{\mu_{\zeta}(\mathrm{x}) \mid \zeta \in \mathrm{EM}\right\}$ is a set of coherence membership functions of $(E M, \vee, \Lambda)$ and $(M, \tau, X)$, if for each concept $\zeta=\sum_{i \in I}\left(\prod_{m \in A_{i}} m\right) \in E M$, $\mu_{\zeta}: X \rightarrow[0,1]$ is defined as follows: for any $x \in X$

$$
\begin{aligned}
\mu_{\zeta}(\mathrm{x}) & =\sup _{\mathrm{i} \in \mathrm{I}}\left(\prod_{\gamma \in \mathrm{A}_{\mathrm{i}}} \mathcal{M}_{\gamma}\left(\mathrm{A}_{\mathrm{i}}^{\tau}(\mathrm{x})\right)\right. \\
\mu_{\zeta}(\mathrm{x}) & =\sup _{\mathrm{i} \in \mathrm{I}}\left(\inf _{\gamma \in \mathrm{A}_{\mathrm{i}}} \mathcal{M}_{\gamma}\left(\mathrm{A}_{\mathrm{i}}^{\tau}(\mathrm{x})\right)\right.
\end{aligned}
$$

\section{Proof:}

$$
\text { Let } \quad \alpha=\sum_{i \in I}\left(\prod_{m \in A_{i}} m\right), \quad \beta=\sum_{j \in J}\left(\prod_{m \in B_{j}} m\right) \in
$$
EM and $\alpha \leq \beta$ in lattice (EM, $\vee, \Lambda$ ). By Theorem 1 , for any $A_{i}(i \in I)$, there exist $B_{h}(h \in J)$ such that $A_{i} \supseteq B_{h}$. Then $A_{i}^{\tau}(x) \subseteq B_{h}^{\tau}(x)$ for any $x \in X$. Thus for any $i \in I$,

$$
\begin{aligned}
\prod_{\gamma \in A_{\mathrm{i}}} \mathcal{M}_{\gamma}\left(\mathrm{A}_{\mathrm{i}}^{\tau}(\mathrm{x})\right) & \leq \prod_{\gamma \in \mathrm{A}_{\mathrm{i}}} \mathcal{M}_{\gamma}\left(\left(B_{h}^{\tau}(x)\right)\right. \\
& \leq \prod_{\gamma \in \mathrm{B}_{\mathrm{h}}} \mathcal{M}_{\gamma}\left(B_{h}^{\tau}(x)\right) \\
& \leq \mu_{\beta}(\mathrm{x}) .
\end{aligned}
$$

which implies that,

$$
\mu_{\zeta}(\mathrm{x})=\sup _{\mathrm{i} \in \mathrm{I}}\left(\inf _{\gamma \in \mathrm{A}_{\mathrm{i}}} \mathcal{M}_{\gamma}\left(\mathrm{A}_{\mathrm{i}}^{\tau}(\mathrm{x})\right) \leq \mu_{\beta}(\mathrm{x})\right.
$$

Thus condition 1 of Definition 8 holds.

Since $\mathcal{M}_{\gamma}(\emptyset)=0$, for any simple concept $\gamma \in \mathrm{M}$ hence condition 2 of Definition 8 holds.

For $x, y \in X, A \subseteq M, \quad \eta=\prod_{m \in A} m \in E M$, if $\mathrm{A}^{\tau}(\mathrm{x}) \subseteq$ $\mathrm{A}^{\tau}(\mathrm{y})$, then for any $\gamma \in \mathrm{A}$,

$$
\left.\mathcal{M}_{\gamma}\left(\mathrm{A}_{\mathrm{i}}^{\tau}(\mathrm{x})\right) \leq \mathcal{M}_{\gamma} \mathrm{A}_{\mathrm{i}}^{\tau}(\mathrm{y})\right) .
$$

This implies that $\mu_{\eta}(\mathrm{x}) \leq \mu_{\eta}(\mathrm{y})$. Furthermore, $\operatorname{since}_{\mathcal{\gamma}}(\mathrm{X})=$ 1 , hence condition 3 of Definition 8 holds. Therefore $\left\{\mu_{\zeta}(\mathrm{x}) \mid\right.$ $\zeta \in E M\}$ is the set of coherence membership functions of $(E M, \vee, \Lambda)$ and $(\mathrm{M}, \tau, \mathrm{X})$. As for each $\gamma \in \mathrm{M}$ and for any $\mathrm{U} \in$ $\mathrm{S}, 0 \leq \mathcal{M}_{\gamma}(\mathrm{U}) \leq 1$ and $\mathcal{M}_{\gamma}(\mathrm{U})=1$, the functions defined by (2.1) or (2.2) are coherence membership functions. In real world applications, the measure $\mathcal{M}_{\gamma}$ can be constructed according to the semantic meaning of the simple concept $\gamma$ and may have various versions depending on the identification of the problem.

In general, $\mathcal{M}_{\gamma}\left(A_{\tau}(\mathrm{x})\right)$ measures the degree of set $A_{\tau}(x)$ supporting the claim : " $x$ belongs to ". The coherence applied to membership functions of fuzzy concepts in $E M$ to denote the membership functions with respect to the semantic interpretations expressed by the fuzzy concepts, the logic relationships among the fuzzy concepts in Axiomatic Fuzzy Set logic systems $(E M, \vee, \Lambda)$. AFS theory presents a far more flexible and potential framework for achieving and representation of human knowledge which is suitable for studying large-scale intelligence systems in real applications.

\section{CONCLUSION}

Axiomatic Fuzzy Set theory proposes a future flexible and powerful framework for acquisition and illustration of human knowledge which is appropriate for studying large-scale intelligence systems in real-time applications. Axiomatic Fuzzy set theory approach is considered as the knowledge representation of the system, it helps in compact with data sets of mixed data type features processed in a uniform way. In addition, AFS theory with the properties of AFS algebra and AFS structures, the membership functions and their logic operations for any model can be achieved commonly based on the original statistical data and by using the axiomatic theory coherence membership function its focal point on an innovative design of rule extraction. The design helps to deal with cases where it meets imbalanced classes. The modification and pruning methods help to generate a echo and efficient fuzzy rule-based classifier.

\section{REFERENCES}

[1] X.D. Liu, The Fuzzy Theory Based on AFS Algebras and AFSStructure, Journal of Mathematical Analysis and Applications217 (1998) 459-478.

[2] X.D. Liu, W. Pedrycz, Q. Zhang, Axiomatic Fuzzy Sets Logic,IEEE International Conference on Fuzzy Systems 1 (2003) 55-60.

[3] X.D. Liu, The Topology on AFS Algebra and AFS Structure,Journal of Mathematical Analysis and Applications 217 (1998)479 - 489.

[4] X.D. Liu, The Fuzzy Sets and Systems Based on AFS Structure,EI algebra and EII algebra, Fuzzy Sets and Systems 95 (1998)179-188.

[5] X.D. Liu, W. Pedrycz, The Development of Fuzzy DecisionTrees in the Framework of Axiomatic Fuzzy Set Logic, Applied Soft Computing 7 (2007) 325-342.

[6] X.D. Liu, W. Wang, T. Chai, The Fuzzy Clustering Analysis Based on AFS Theory, IEEE Transactions on Systems, Man and Cybernetics: Part B 35 (5) (2005)

[7] Yan Ren, Mingli Song, and Xiaodong Liu, New Approaches to the Fuzzy Clustering Via AFS Theory, International Journal of Information and Systems Sciences, Volume 3, Number 2, Pages 307-325(2007)

[8] X.D. Liu, W. Liu, The Framework of Axiomatic Fuzzy Sets Based Fuzzy Classifiers, Journal of Industrial Management Optimization 4 (3) (2008) 581-609.

[9] L. Wang, X. Liu, Concept Analysis via Rough Set and AFS Algebra, Information Sciences 178 (2008) 41254137.

[10] X.D. Liu, W. Pedrycz, T.Y. Chai, M.L. Song, The Development of Fuzzy Rough Sets with The Use of Structures and Algebras of Axiomatic Fuzzy Sets, IEEE Transactions on Knowledge and Data Engineering 21 (3) (2009) 443-462.

[11] X.D. Liu, Q.L. Zhang, The Fuzzy Cognitive Maps Based on AFS Fuzzy Logic, Dynamics of Continuous, Discrete and Impulsive Systems, Series A: Mathematical Analysis 11 (2004) 787-796.

[12] X.D. Liu, W.Q. Liu, Credit Rating Analysis with AFS Fuzzy Logic, Lecture Notes in Computer Science (LNCS) 3612 (2005) 1198-1204.

[13] X.D. Liu, W. Pedrycz, Axiomatic Fuzzy Set Theory and Its Applications, Springer Verlag, Heidelberg, Germany, 2009.

[14] X.D. Liu, T.Y. Chai, W. Wang, W.Q. Liu, Approaches to the representations and logic operations for fuzzy concepts in the framework of axiomatic fuzzy set theory 
I, II, Information Sciences 177 (2007) 1007-1026, 10271045.

[15] X. Liu, The Structure of Fuzzy Matrices, Journal of Fuzzy Mathematics 2 (1994) 311-325.

[16] X. Liu, A new mathematical axiomatic system of fuzzy sets and systems, Journal of Fuzzy Mathematics 3 (1995) 559-560.

[17] X.D. Liu, Two algebra structures of AFS structure, Journal of Fuzzy Mathematics 3 (1995) 561-562.

[18] X. Liu, Q. Zhang, AFS fuzzy logic and its applications toFuzzy information processing, Dongbei Daxue Xuebao 23 (4) (2002) 321- 323 (in Chinese).

[19] X. Liu, Q. Zhang, The EI algebra representations of fuzzyconcept, Fuzzy Systems and Mathematics 16 (2) (2002) 27-35.

[20] X. Liu, K. Zhu, H. Huang, The representations of fuzzy concepts based on the fuzzy matrix theory and the AFS theory, in: Proceedings of IEEE International Symposium on Intelligent Control, Houston, TX, USA, 2003, pp. 1006-1011.

[21] K.H. Kim, Boolean Matrix Theory and Applications, Marcel Dekker, New York, 1982.

[22] X. Liu, X. Feng, Witold Pedrycz, Extraction of fuzzy rules from fuzzy decision trees: on Axiomatic fuzzy sets approach, Data\& knowledge Engineering 84 (2013), 125.

[23] Y. Zhang, D. Liang, S. Tong, On AFS algebra Part I, Information Sciences 167 (2004) 263-286.

[24] Y. Zhang, D. Liang, S. Tong, On AFS algebra Part II, Information Sciences 167 (2004) 287-303.

\section{AUTHOR PROFILE}

Lakshmi Ramani Burra is a research scholar in the department of Computer Science and Engineering at GITAM University,Visakhapatnam, Andhra Pradesh, India. She received her Master degree in Computer Science and Engineering from Acharya Nagarjuna University 2008. Currently she has been working as Assistant Professor, in the Department of Computer Science \& Engineering, Prasad V. Potluri Siddhartha Institute of Technology, Vijayawada, since 2008. Her research interest is data mining and machine learning

Padmaja Poosapati is an Associate Professor in Department of Information Technology at GITAM University, Visakhapatnam and Andhra Pradesh, India. She received her Master degree in Computer Science and Engineering from Andhra University in 1999 and Ph.D. degree in Computer Science and Engineering from Andhra University 2010. Her Current Research interests include Data Mining, Graph Mining, Artificial Intelligence, and Image Processing. 